\title{
Application Study of Vascular Interventional Robotic Mechanism for Remote Steering
}

\author{
Zengmin Tian*, Bo Jia, Wangsheng Lu and Rui Hui
}

Zengmin Tian, MD Department of Neurosurgery, Navy General Hospital 6 Fucheng Road CN-100037 Beijing, P.R. China

\begin{abstract}
Background: Recently, robotic systems have been introduced as a useful method for surgical procedures. But in the field of vascular interventional therapy, the development of robotic system is slower.

Objective: The purpose of the study is to verify the reliability and safety of vascular interventional robotic system used in angiography, by the way of in vitro preliminary experiments and animal experiments.

Method: The approach is to employ a proprietary vascular interventional robot system to complete glass vessel models and animal angiogram experiments. This robot system consists of a console port (remote steering system), an assistant port (propelled and rotation system) and a hydraulic fixing device, upon which surgeons control remotely to make go forward and rotate in the glass vessel models and animal vessels, on the 3D operation interface. Consequently, the operation time and success rate are counted and evaluated.

Result: In the glass vessel model experiments, the_Catheter can enter various kinds of vessel models with inside diameter length greater than $3 \mathrm{~mm}$ and angle less than $90^{\circ}$. In the animal (adult dogs) experiments, surgeons can accomplish smoothly the angiogram of the renal artery, the vertebral renal and the arteria carotis communis, without any complications of surgery.

Conclusion: The angiogram by using vascular interventional robot system is safe and reliable. Surgeons can finish the angiogram in part by remote operation, and the result of angiogram can meet a number of simple expectations. However without wire control and force feedback systems, the applicability of this kind of robot system is not flexible enough and need to be improved in the future.
\end{abstract}

Keywords: Robotic, vascular interventional, remote steering, animal experiments.

\section{INTRODUCTION}

Cardiovascular disease serving as a major reason of death currently is the world's fastest-growing disease with high morbidity, disability and mortality rates. Since the eighties in the last century, interventional operation has widened clinical applications due to its merits including minimal injury, quick postoperative recovery and favorable operative effectiveness and so on. With respect to vascular application, at present, surgeons often insert a catheter and a guide wire into formal vein and artery in the interventional operation, by which the catheter and guide wire reach the target treatment position through handling them by a way of turning or pushing or other movements to carry out various kinds of treatments under X-rayed exposure and navigation. However, some disadvantages of interventional treatment constraint its development. Firstly, although many protective devices have been adopted, long time exposure and great number of operations for doctors result in radiation injury. Secondly, bootstrap images that just can display vascular stereochemical structure predominately are 2D (2Dimensional) blood vessel image formation instead of 3D.

*Address correspondence to this author at the Department of Neurosurgery, Navy General Hospital 6 Fucheng Road CN-100037 Beijing, P.R. China; Tel: +86 106878 0358; Fax: +86106878 0358;

E-mail tianzengmin@sina.com
Thirdly, instability of manual operation probably reduces achievement ratio of operation and even can result in vascular and organic injury. On the other hand, robotics has been applied to urinary and abdominal cavity operations as an original technological means [1 2], leading to vascular interventional robot gaining more and more attention. Currently, there are several commercial interventional robots that still having many disadvantages including specified catheter and guide wire, complicated training, pricey costs and so on. Domestic research is still vacant. The present study inducts a vascular interventional robot with independent intellectual property right which is employed to interventional treatment and to carry out empirical application study. The aims of this study contain three parts. First, it would avoid radiation injury through remote steering. Second, robot can handle the catheter stably and accurately to avoid the injury induced by manual operative fault. Third, it would rebuild three-dimensional signature via multi-angular two dimensional image formation to make operative planning convenient, in order to guide operative procedure and reduce difficulty.

\section{MATERIALS AND METHODS}

\subsection{Interventional Robot Systems}

A vascular interventional robot contains three main components, including propelled and rotation system, image 
navigation and management system ( remote steering system ), and a hydraulic fixing device.

Propelled and rotation system ensures that a doctor can turn catheter and guide wire and push or pull (forward or backward) to control its direction by analyzing the movements of interventional operative procedure. The system close to the patient simulates the movement of human hands, and then fulfils straight forward and backward or contorted movements of catheter through revolving the gear wheel.

Image navigation and management system is composed of operating handle and main control station. Doctors order mechanical propulsion system close to the patient through operating handle to control the catheter movement. Visual orientation system, DSA (i.e., digital subtraction angiography) image correction software and vascular threedimensional reconstruction software based on multi-angular DSA images constitute an image reconstruction system. Visual orientation system tracks two kinds of trace labeling which have fixed geometrical relationship with DSA image formation system to determine the space coordinates of Carm $X$ light source and image intensifier. DSA images as Xray subtraction images are applicable to navigate and orientate them whereas distortion correction is in need to ensure the precision of operative navigation and to avoid distortion and deformation. Finally, they reconstruct threedimensional vascular stereoscopic images according to the multi-angular DSA images. It is possible to integrate two dimensional projections into reconstructed three-dimensional images to implement real-time orientation and navigation of catheters.

The hydraulic fixing device contains five flexible sap pressure joint and can be fixed in any angle at any operation table. Doctors can adjust robotic fixed position according to coursers and the direction of vascular puncture.

\subsection{Vascular Model Experiment}

At first, vascular model experiment is carried out to measure the basic function of robot system. This experiment mainly is executed to investigate the remote controlled robot in controlling ductal movement in a glass vascular model and measuring characteristics of the ductal speed, rotated angle and so on. Meanwhile, it implementes threedimensional reconstruction of glass vascular model. The glass vascular model imitating human blood vessels contains simulated vascular morphous with an inner diameter of $2 \sim 10 \mathrm{~mm}$ and many branches with $30 \sim 90^{\circ}$ bifurcation angles between daughter branches and their parent blood vessels. The viscosity in a glass vascular model approximates the transparent solution of human blood. In our experiments, first, a $4 \mathrm{~F}$ catheter is entered vascular model through arteriopuncture sheath, whereby basic functions are measured including its speed, maximum angle of rotation in an euthyphoria state. Then under DSA operating condition, remote controlled robot handles the catheter to reach vessels of different inner diameters, different angles of terminal branch of vascular model and counts the achievement ratio and required time. Finally, contrast agent is injected into the vascular model that takes $30^{\circ}$ of left anterior oblique and $30^{\circ}$ of right anterior oblique of $\mathrm{C}$-arm to visualize image in order to reconstruct three-dimensional images of vascular model. Thereafter, ductal movement in vascular model is monitored and guided according to integral threedimensional images. At last, errors between the operative schedule and the practical operation are statistical analyzed.

\subsection{Animal Experiment}

Animal experiment has been carried out under DSA operating condition after preliminary mastering the basic function and operational approach as demonstrated in Fig. (2).

\subsubsection{Subject}

Ten experimental dogs with an average age of eighteen months of both sexes (body weight from $10 \mathrm{~kg}$ to $15 \mathrm{~kg}$ ) were maintained in Peking University Health Science Center. Operative equipments consist of conventional incision suture package, a $4 \mathrm{~F}$ single-bending catheter, and ioversol as a contrast agent. Model of DSA isGE LCV mode named digital vascular subtraction X-ray apparatus.

\subsubsection{Methods}

Dogs had been fasted overnight for 12 hours and anesthetized by a mixture of ketamine with a dosage of $5 \mathrm{mg} / \mathrm{kg}$ and a sumianxin injection with a dosage of $0.08 \mathrm{ml} / \mathrm{kg}$. Since tracheal intubation provided constant low flow oxygen inhalation, sodium Chloride $(500 \mathrm{ml})$ with cidomycin $(200,000$ units) was persistent intravenous injected. The ECG monitor was provided at experimental sessions. The experimental dogs were supinated and fixed at an operation table with sterilized surface of right fold inguen after anesthetizing and the part related to extreme obvious pulse of femoral artery was cut. We adopted modified Seldinger measure to carry out right femoral artery puncture with the exposure of femoral artery under euthyphoria condition. Fixing interventional robot made the forward direction of catheter to fit the courser of femoral artery. Rectal cauda was connected with hyperbaric contrast medium injector.

The operator controlled robot performed tele-operation under perspective guidance and impelled catheter to the abdominal aortas of those experimental dogs approximately at an inferior border of double ribs level through rotation and pushing or pulling. Images were visualized at $30^{\circ} \mathrm{of}$ left anterior oblique and $30^{\circ}$ of right anterior oblique of $\mathrm{C}$-arm according to two dimensional projections and the computer would produce three-dimensional vascular stereoscopic image, including double sides renal artery, celiac trunk, superior mesenteric artery and splenic artery. This can determine debouch direction and the angle of a target blood vessel in the main blood vessel. The catheter was inserted into debouch of right renal artery while right renoarteriography was carried out under three-dimensional images navigation. Angiography effectiveness of extracalvarium segments of vertebral artery and carotid artery were satisfactory compared with intracalvarium segments of carotid artery. All the experiments were accomplished by interventional doctors who had at least 3 years of experience and carried out more than 100 cases of interventional operations by a way of conventional operative measures and timing (angiography time was excluded). It is necessary to extract puncture sheath and use pressure dressing after operation. 


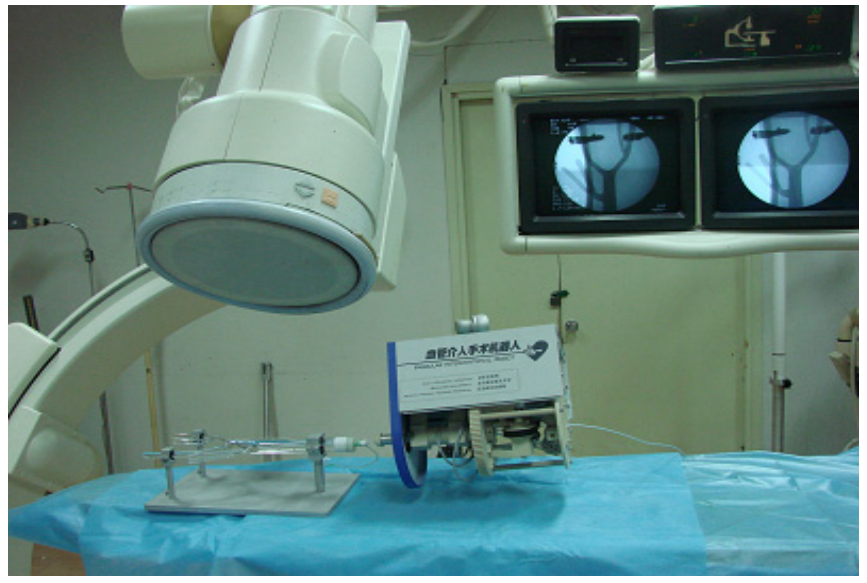

Fig. (1). Vascular model experiment.

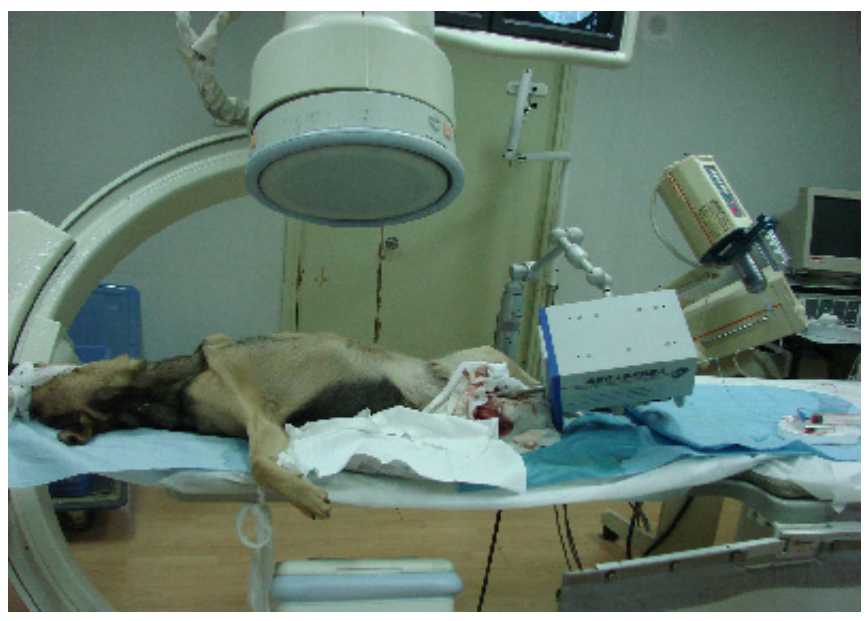

Fig. (2). Animal experiment.

\section{RESULTS}

The propulsion speed is $1.3 \mathrm{~cm} / \mathrm{s}$ and it can implement any angle of ductal rotation in preliminary experiment of glass vascular model in vitro. The interventional robot control catheter to branch of vascular model whose inner diameter is more than or equals to $3 \mathrm{~mm}$ and includes an angle being less than or equals to $90^{\circ}$. Table 1 shows the achievement probability and required time after repeating ten times of the movements. practical model. Thus, the mechanical function of vascular interventional robot can meet interventional needs and accurately establish three-dimensional images, while providing real-time monitoring of the position and movement of a catheter.

All experiments were carried out successfully in the animal experiments. The doctors can guide and telemanipulate the robot to control $4 \mathrm{~F}$ catheter move to renal artery, aortic arch, left vertebral artery and can accomplish angiography smoothly after establishing three-dimensional vascular model. The doctors repeated these movements as described. The average required time was $12.2 \mathrm{~min}$ with an average prolonged time about $10 \sim 15 \mathrm{~min}$. This trend can be attributed to two aspects. First, operators spent time on grasping the mechanical skills. Second, the mechanical propulsion of the interventional robot with constant pace lacked of speed regulation.

Ten postoperative dogs returned to normal after palinesthesia without complications. In addition to insertion and extraction of arterial sheath, doctor can telecontrol robot to carry out the experiment without radioactive exposure while performing robotic operation. Animal experiments have demonstrated that a robot can accomplish adult canine renal arteriography, aortic arch and vertebral arteriography smoothly. Although the required time was slightly longer than manual operation, there were not any complications.

\section{CONCLUSION}

Vascular interventional operation has been widely applied to treat various diseases by giving advantages of minimal injury, favorable effectiveness and quick recovery. However, conventional interventional operation also confronts a series of difficulties. With wide application of robotics in the medical field, making robot available in an interventional operation attracts an increasing attention [3,4]. At present, Hansen medical company (http://www.hansen medical.com/company) has commercialized interventional robots. Moreover, studies indicate that this system can reduce radioactive exposing time and injury effectiveness.

However, a number of other studies have reported that the incidence rate of complication in interventional robotic systems is higher than that of conventional interventional operations. Meanwhile, the system requires specified

Table 1. Achievement Probability and Required Times for the Nine Experiments

\begin{tabular}{|c|c|c|c|c|c|c|c|c|c|}
\hline Vascular Model & $30^{\circ} 8 \mathrm{~mm}$ & $30^{\circ} 5 \mathrm{~mm}$ & $30^{\circ} 3 \mathrm{~mm}$ & $60^{\circ} 8 \mathrm{~mm}$ & $60^{\circ} 5 \mathrm{~mm}$ & $60^{\circ} 3 \mathrm{~mm}$ & $90^{\circ} 8 \mathrm{~mm}$ & $90^{\circ} 5 \mathrm{~mm}$ & $90^{\circ} 3 \mathrm{~mm}$ \\
\hline Achievement Probability & $100 \%$ & $90 \%$ & $70 \%$ & $100 \%$ & $90 \%$ & $60 \%$ & $80 \%$ & $60 \%$ & $50 \%$ \\
\hline Time (s) & 7.3 & 8.1 & 11.4 & 7.9 & 10.4 & 13.5 & 9.8 & 14.6 & 19.0 \\
\hline
\end{tabular}

The data suggest that the included angles between branch and main blood vessels and required time increase with the decrease of inner diameters of glass branches. Likewise, the achievement ratio decreased. This is consistent with the common law of manual angiography. Reconstructed threedimensional vascular model can induce catheter to move to a specified branch exactly with less than $0.5 \mathrm{~cm}$ practical error under DSA operating conditions. Three-dimensional reconstructed vascular model is consistent with the form of catheter, expensive expenditure and complicated training. On the other hand, teleoperative robot exploited by center of Haifa cardiac disease in Israel [5] has been applied to cardiovascular experiment, but interventional treatment for other diseases is poorly understood. Domestic research is one kind of embryonic research and still in the theoretical stage at present. We have applied three-dimensional directional robots to accomplish 3300 cases of operations and carried out remote teleoperation robotic surgeries. 
Beijing University of Aeronautics and Astronautics and Naval General Hospital continue to cooperate with each other and design vascular interventional teleoperation robotic specimen successfully through analyzing technological highlights and operative standards for vascular interventional operations. In an interventional robot system, an image navigation system can reconstruct three-dimensional vascular image according to multi-angular plane vascular projections whereby doctors can order teleoperation mechanical propulsion system to make catheter reach specified blood vessel in time and accomplish angiography smoothly according to three-dimensional images.

This preliminary study has demonstrated the feasibility, safety and effectiveness of vascular interventional teleoperation robot and can serve as a foundation, paving the way for a robot to be applied to vascular interventional clinical operation.

On the other hand, interventional robot also has some deficiencies. In the conventional interventional operation, doctors can make catheter move to a diseased region through inducement of the guide wire to overcome the difficulties including complex anatomic structure, large anfractuous angles and pathological blood vessels. In present study, because of the lack of guide wire control system, it is difficult for vascular interventional robot system to reach the branches with larger anfractuous angle and smaller inner diameter, which therefore increases running time in a number of operations carried out in this investigation. Based on the resistance, doctors can perceive the terminal pressure of catheter and guide wire to reduce the vascular and organic injury when they impelled catheter. Although we have studied on ductal terminal baroreceptor, a teleoperation robotic system has not gained any practical application yet because of the shortage of feedback systems. It is necessary to induct both force feedback system (haptics) and guide wire control system into the robot, leading to the vision of a robot system of vascular interventional being applied to a clinical interventional operation becomes a reality.

\section{ACKNOWLEDGEMENT}

None declared.

\section{CONFLICT OF INTEREST}

None declared.

\section{REFERENCE}

[1] Mack MJ. Minimally invasive and robotic surgery. JAMA 2001; 285: 568-72.

[2] Da 1, Zhang D, Wang T. Overview of the vascular interventional robot. Int J Med Robot 2008; 4: 289-94.

[3] Saliba W, Cummings JE, Oh S, et al. Novel robotic catheter remote control system: feasibility and safety of transseptal puncture and endocardial catheter navigation. J Cardiovasc Electrophysiol 2006; 17: 1102-5.

[4] Riga CV, Bicknell CD, Wallace D, et al. Robot-assisted antegrade in-situ fenestrated stent grafting. Cardiovasc Intervent Radiol 2009; 32: 522-4.

[5] Anchorage, Alaska, Advanced surgical service robotics in the European Union 6th and 7th Framework Programs, 2010 IEEE International Conference on Robotics and Automation, 2010. 